\title{
Managing patients in the emergency department with mental health and substance use disorders
}

\author{
Kimberley DuBose, Johnie Leonard, Melissa Graham \\ Houston Methodist Hospital, Houston, Texas, USA
}

Received: September 27, 2018

Accepted: October 24, 2018

Online Published: August 23, 2019

DOI: $10.5430 /$ jnep.v9n12p35

URL: https://doi.org/10.5430/jnep.v9n12p35

\begin{abstract}
With approximately 3\% of the Houston Methodist Hospital Emergency Department's (ED) 2017 annual volume presenting with resource-intensive psychiatric conditions, a 6-prong approach was applied to address the growing area of concern on how to best manage this unique population safely and efficiently through the provision of high quality care. This approach included (1) the provision of dedicated care space, (2) placement of a trained team of providers and clinical staff, (3) contracting with a third-party, rapid-screen care team, (4) application of new technology, (5) instilling a partnership with the ancillary team, and (6) extending care after the hospital stay for better management of the longevity of the patients' medical issues. Through these efforts, the overall time from ED arrival to ED departure for psychiatric patients who were discharged was reduced by $36 \%$. In addition, the admit decision time to ED departure time for psychiatric patients was reduced by $30 \%$ from 2016 to the third quarter of 2017. Additionally, the number of violent patient incidents in the ED mental health unit was reduced to zero from 2016 to the third quarter of 2017 , a number that is holding to date. Via the presence of heightened security measures, approximately $50 \%$ fewer security dispatches were requested in 2018 than in 2017. This is even more profound when considering the $9 \%$ growth in overall ED patient volume over the same time period. Thus, through the application of a multifaceted approach to the care of patients with mental health and substance use disorders presenting to the Houston Methodist Hospital ED there was an observed significant positive effect. Continued diligence to this topic in addition to further expanded resources are needed in both the community and clinical setting to mitigate the negative cycle of patients unnecessarily returning to the hospital or landing in jail that currently exists.
\end{abstract}

Key Words: Mental health, Substance use disorders, Emergency department

\section{INTRODUCTION}

In 2016, the Houston Methodist Hospital Emergency Department (ED) met the call of the community to better address the managing of patients with mental health and substance use disorders through a series of process improvements, with positioning of key resources and personnel in addition to the placement of new technology. The specific goal of the project was to reduce the length of stay (LOS) of this patient population in the ED. The design included the facilitation of quicker provider interactions with patients upon the patients' arrival to the hospital as well as more efficient dispositioning of the patients after assessment to improve the medical management of the psychiatric patient. A context of a safe, violent-free environment for the patient and care team provided the foundation for the undertaking.

With approximately $3 \%$ of the Houston Methodist Hospital ED's 2017 annual volume presenting with resource-intensive psychiatric conditions, a number that is growing with every

*Correspondence: Kimberley DuBose; Email: kdubose @ houstonmethodist.org; Address: Houston Methodist Hospital, Houston Texas, USA. 
passing day, and in consideration of the limited care offerings in the greater Houston area, Houston Methodist Hospital applied a 6-prong approach. This approach included (1) the provision of dedicated care space, (2) placement of a trained team of providers and clinical staff, (3) contracting with a third-party, rapid-screen care team, (4) application of new technology, (5) instilling a partnership with the ancillary team, and (6) extending care after the hospital stay for better management of the longevity of the patients' medical issues.

Through these efforts, the admit decision time to ED departure time for psychiatric patients was reduced by $30 \%$ from 2016 to the third quarter of 2017. In addition, the overall time from ED arrival to ED departure for psychiatric patients who were discharged was reduced by $36 \%$. Additionally, the number of violent patient incidents in the ED mental health unit was reduced to zero from 2016 to the third quarter of 2017 , a number that is holding to date. Via the presence of heightened security measures, approximately $50 \%$ fewer security dispatches were requested in 2018 than in 2017 . This is even more profound when considering the $9 \%$ growth in overall ED patient volume over the same time period.

Thus, the multifaceted approach to the care of patients with mental health and substance use disorders presenting to the Houston Methodist Hospital ED was perceived to have had a significantly positive effect.

\section{BACKGROUND AND SIGNIFICANCE}

According to the Agency for Healthcare Research and Quality (2010), an estimated 1 in 8 emergency room visits involves a mental health and/or substance use condition. In recent years, EDs in Houston have seen a greater uptick than average in mental health patients entering their doors owing to the decrease in inpatient psychiatric bed resources in the local area. Currently, there are only 2 inpatient psychiatric care options, one with only 274 acute-care beds and the other with 32 beds, including 12 in its emergency center, to serve Houston's population of approximately 7 million. ${ }^{[1,2]}$

In addition, there is an overall lack of providers for the care of psychiatric patients. ${ }^{[3]}$ Much of the onus for quick and ready resources for this patient population lies with hospital EDs to provide a safety net for patients seeking care. Many of the patients arriving to the ED do so in an unstable and agitated state with the need for prompt attention from ED physicians and staff. The pressure to care for this populous in a fitting manner while maintaining the safety of the providers and staff who care for them is a critical consideration.

Furthermore, with very little support currently available to continue care after discharge, an undesirable sequence of events is seen, with patients being discharged only to end up returning for subsequent treatment or being arrested and sent to jail. The Harris County Jail calculates that it houses more psychiatric patients than all the state hospitals combined. ${ }^{[1]}$ With a resulting public health emergency due to long waits for limited health care resources, Texas lawmakers and health care providers alike are calling for innovative behavioral health care programs and new ways to connect patients to psychiatric professionals remotely. ${ }^{[4]}$

To respond to this call to action and help address the community's needs, Houston Methodist Hospital primed itself for a change in its approach to the care of this assemblage from arrival to the ED and, in partnership with community resources, to help transition patients back into the public with the support they need to be successful.

\section{STRATEGY AND IMPLEMENTATION}

To address the gaps in emergency medicine related to the population of patients with mental health and substance use disorders, Houston Methodist Hospital implemented a 6pronged approach.

\subsection{Dedicated space}

First and foremost, a dedicated physical space was established within the ED that promoted the safety of the patient and the care team. Where once care for this patient population was conducted in 4 curtained rooms and 1 isolation room, the ED was expanded in 2016 to a secured 6-room unit where all rooms were primed for isolation as needed. The space was designed with a focus on the needs of the patients who would occupy it as well as the clinical staff who would care for them. An architecture firm was contracted to aid in the research of best practices in devices and design to support an area of this type, while maintaining the high standard of ethical patient treatment. Special standards were adhered to, such as special mirrors, soap dispensers, and sinks, for private psychiatric-safe patient rooms and bathrooms. Reinforced walls and encased medical gas units and televisions provided extra-durable protection for the patient and the equipment used in their care. Certain design elements, such as anti-ligature doors and door handles were evaluated and installed.

In addition, staff technology for room monitoring was implemented, allowing a mental health technician to fully monitor all in-room activity at a private central station. Because one staff member could monitor more than one patient at a time, rather than needing one staff member to watch one patient in a room directly, disbursement of staff resources to address other patient care needs was maximized. To further ensure the safety of patients and staff, duress buttons were installed. These buttons located at the nurses station 
were provided to allow clinical staff to call for assistance should security or additional clinical staff be needed to address patients who presented safety concerns. This system supplemented armed police officers who were on-site 24-7 stationed in the psychiatry-enabled unit, as well as at the entry of the ED, along with security monitors that projected the views of all public areas for monitoring by security and staff.

Through scrutiny of the intended flow of the patients through the unit and research into best practices in mental health and substance use disorder clinical care area functionality, the 6-bed area was well designed with the patients it served and the staff who cared for them in mind - and safety being top of mind.

\subsection{Trained team of providers and clinical staff}

With the facility design optimized, Houston Methodist Hospital focused on the clinical care team. The hospital hired a highly trained team of mental health technicians, nurses, and midlevel staff to aid in the facilitation of the patient care plan. By ensuring that staff were prepared for the level of care needed for this patient population, instances of adverse or violent behavior could be mitigated through the use of learned techniques. Psychiatric-specific documentation, both court and clinically based, as well as hands-on care was taught to ensure that the clinical staff carried out the care plan efficiently to maneuver the patient either to their final admission location or to a stable discharge in a timely fashion. Expediting patients to their next destination in turn made room for the next patient in a quick and efficient manner.

These subject matter experts also shared their knowledge and applied their science by helping to train the rest of the ED staff who may not focus directly on this targeted patient population. For example, the mental health technicians were involved in ensuring that staff in triage who checked patients in knew how to best identify and manage patients who presented with suicidal ideations or with substance misuse, prepping them for care on the unit through gowning and removal of personal items, to ensure that the patient and those around them were kept safe and secure. Another example was with the topic of human trafficking. Staff from the ED mental health team sit on citywide committees and not only aid in the development of best practices at large but also bring that knowledge back to the unit to be shared with the department's clinical staff.

\subsection{Third-party, rapid-screen care team}

To further bolster the care given to the patients with mental health and substance use disorders presenting to Houston Methodist Hospital, a rapid-screen team was put into place through a third-party contract. This team was responsible for partnering with the on-site clinical team to facilitate treatment and placement of the patient either internally or externally. Contracted in May 2017, the resource saw an average of 150 patients per month in the second half of 2017. With a response goal of 90 minutes from the time called to on-site screening of the patient, the team averaged less than 50 minutes per month for this same time frame. Commonly, they are seen on the unit for several hours a day. Because they know the ins and outs of the process to get patients placed in care facilities and the paperwork needed to support this endeavor, their partnership has proven invaluable in reducing the LOS in the hospital for the patients they serve.

\subsection{Use of technology}

Given the American Psychiatric Association's stance that "telemedicine in psychiatry, using video conferencing, is a validated and effective practice of medicine that increases access to care," Houston Methodist Hospital implemented its use to supplement the limited provision of psychotherapy providers in the area. ${ }^{[5]}$

Telemedicine, or an interactive audio and video telecommunication system that permits real-time interaction between a remotely located psychiatrist and the patient at the hospital, was implemented in June 2017. The process started with on-site clinical staff scheduling an appointment for the psychiatric medical professional to talk to the patient via a video stream app on an iPad mobile digital device (Apple Inc) to assess the patient and drive the care plan. The nurse would be involved in the call with the patient and provider to hear the plan of care and subsequently carry it out as ordered.

The ED physician on-site further collaborated with the psychiatrist and on-site mental health team to ensure that the patient was medically managed in a successful manner. The mental health providers, in addition to providing real-time critical care updates, conducted quality rounds with the ED physician each shift to review the treatment plan and ensure alignment of intent. This step proved critical to managing comorbidities that this patient population often presented with and ensured that both the mental and physical attributes, like chronic disease, were addressed for better outcomes through the provision of "whole-person" care. ${ }^{[6]}$

The telemedicine service averaged 16 consults per month in the last 6 months of 2017 and promoted expedited care for patients within the ED. As seen with many studies on the topic, such as Egede et al., ${ }^{[7]}$ no significant difference in patient satisfaction or treatment credibility was seen. In fact, patients tended to like the proffered option as it furthered the treatment of their condition in a more timely fashion than 
waiting for a provider to be available in person.

\subsection{Partnership with ancillary team}

To supplement this care by the clinical staff, a multidisciplinary approach was advanced by including ancillary departments like spiritual care, social work, case management, and pharmacy. Keeping with its philosophy of caring for the patient in terms of mind, body, and soul, the spiritual care service implemented a structured rounding program to promote the patient's holistic well-being and offer further support in the care process. It is through this methodology that the patient's ethical rights and core needs are kept at the forefront and supported not only by the clinical staff but by all available resources at hand.

Social work and case management provided aid in helping the patient with the care continuation once released from the hospital. This came in the way of offering help with items such as securing shelter, providing clothing if needed, and supporting in follow-up clinical appointments and connections with support groups.

The ED pharmacy staff, in tandem with the provider and mental health clinical care team, worked diligently to complete medicine reconciliations and provide expedited assistance to patients who had fallen off their medicinal protocols. The high cost of some of the drug options that the patients were prescribed created barriers for the patients staying constant with needed dosages. Thus, these reviews offered an opportunity to provide a more affordable option as well as to encourage the patient to resume medications that they may have self-selected to discontinue.

\subsection{Continuation of care after the hospital stay}

To extend the care continuum beyond the walls of the hospital and ensure that the patient population could continue the path on which they were set, Houston Methodist Hospital established an outside connection in the treatment of patients with substance use disorders. This partnership with an ex- ternal sobering center was initiated with patients on-site at the hospital and continued as the patients stepped outside the hospital doors. Through this seamless transition, accelerated compulsory care was provisioned to patients with substance use disorders needing assistance. This was a critical step to break the cyclic burden on the health care system, allowing for continued and long-term support of patients in a community-based setting. A new offering in 2017 for the Houston Methodist Hospital patient population, it is already being well received both by staff caring for the patients and by the patient themselves.

\section{EVAlUATION AND OUTCOMES}

Approximately 3\% of the Houston Methodist Hospital ED's annual volume for 2017 had an affiliated psychiatric diagnosis. This translated into 1,189 patients visits of this type. To assess the impact of these endeavors on this population, the Houston Methodist Hospital measured several metrics of influence.

First, given the hospital's policy of zero tolerance of workplace violence, which states that "any behavior that interferes with the ability of Houston Methodist physicians and staff to effectively carry out their duties is considered disruptive and will be addressed appropriately", it is necessary to address whether these actions had an impact in this arena. This definition of inappropriate behavior includes both verbal and physical assault and abuse. To this end, the number of violent patient incidents in the ED mental health unit was reduced to zero from 2016 to the third quarter of 2017, and this number has held to date. In addition, via the presence of heightened security measures, there have been almost $50 \%$ fewer security dispatches requested in 2018 than in 2017. This is even more profound considering the $9 \%$ growth in ED patient volume over the same time period. The number of security dispatches to the ED from February 2017 through April 2018 is shown in Figure 1.

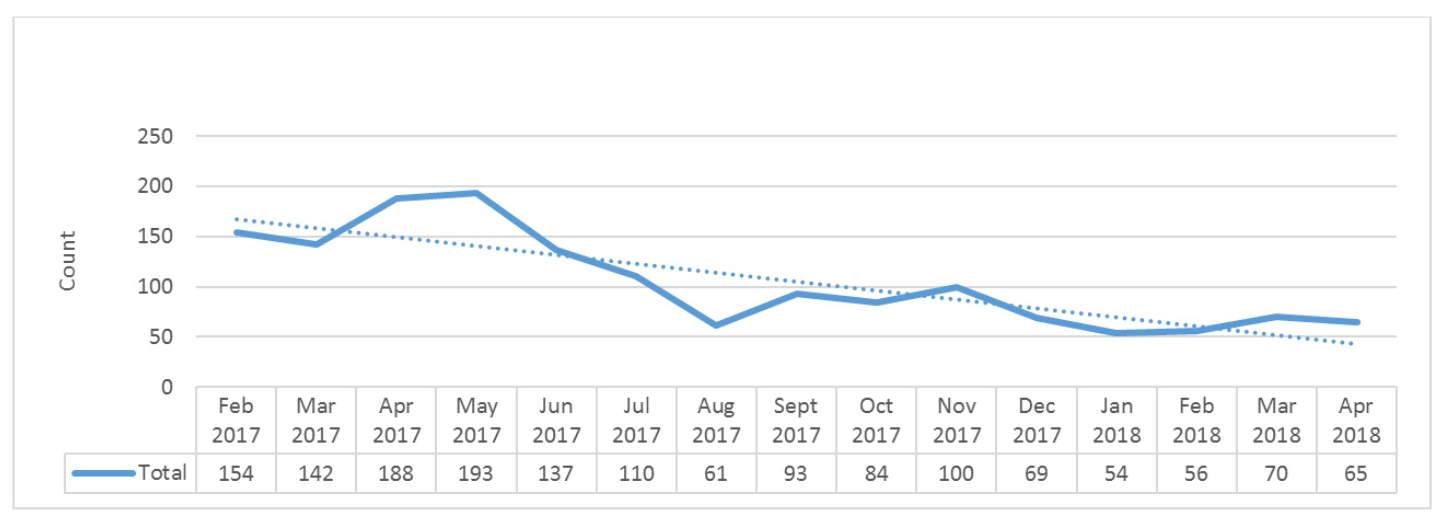

Figure 1. Count of Security Dispatches to the ED 
Impact on LOS for this patient population was also of interest with the desire to lessen the time spent in the hospital setting by reducing the time it takes to get the patient before the provider and establish the appropriate disposition for the care visit. Through utilization of knowledgeable on-site staff, partnership with the rapid-screen care team and psychiatric telemedicine services, the admit decision time to ED departure time for psychiatric patients was reduced by $30 \%$ from
2016 to the third quarter 2017 (see Figure 2). In addition, the overall time from ED arrival to ED departure for discharged psychiatric patients was reduced by $36 \%$ (see Figure 3 ). Thus, the multifaceted approach to the care of patients with mental health and substance use disorders presenting to the Houston Methodist Hospital ED was observed to have had a significantly positive effect.

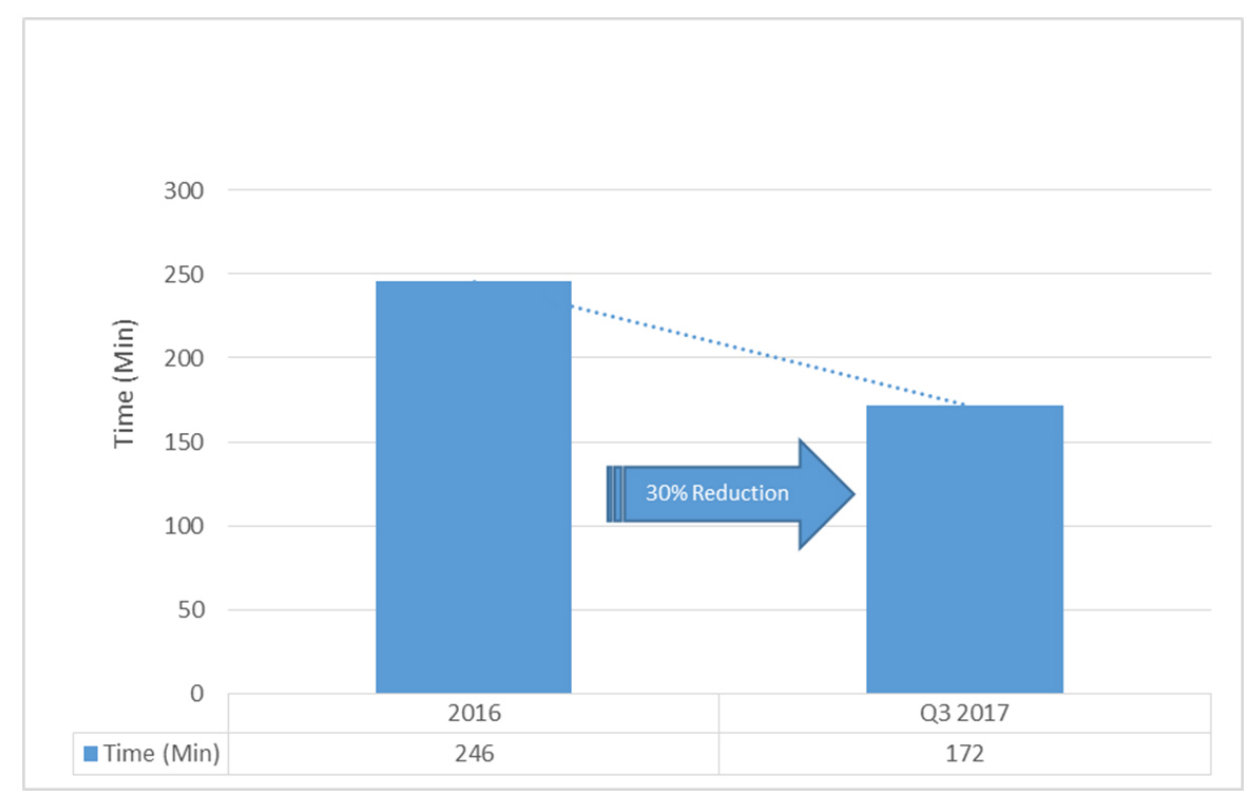

Figure 2. Admit Decision Time to ED Departure Time for Psych Patients

*Source: CMS ED-2c - Admit Decision Time to ED Departure Time - Psychiatric Patients

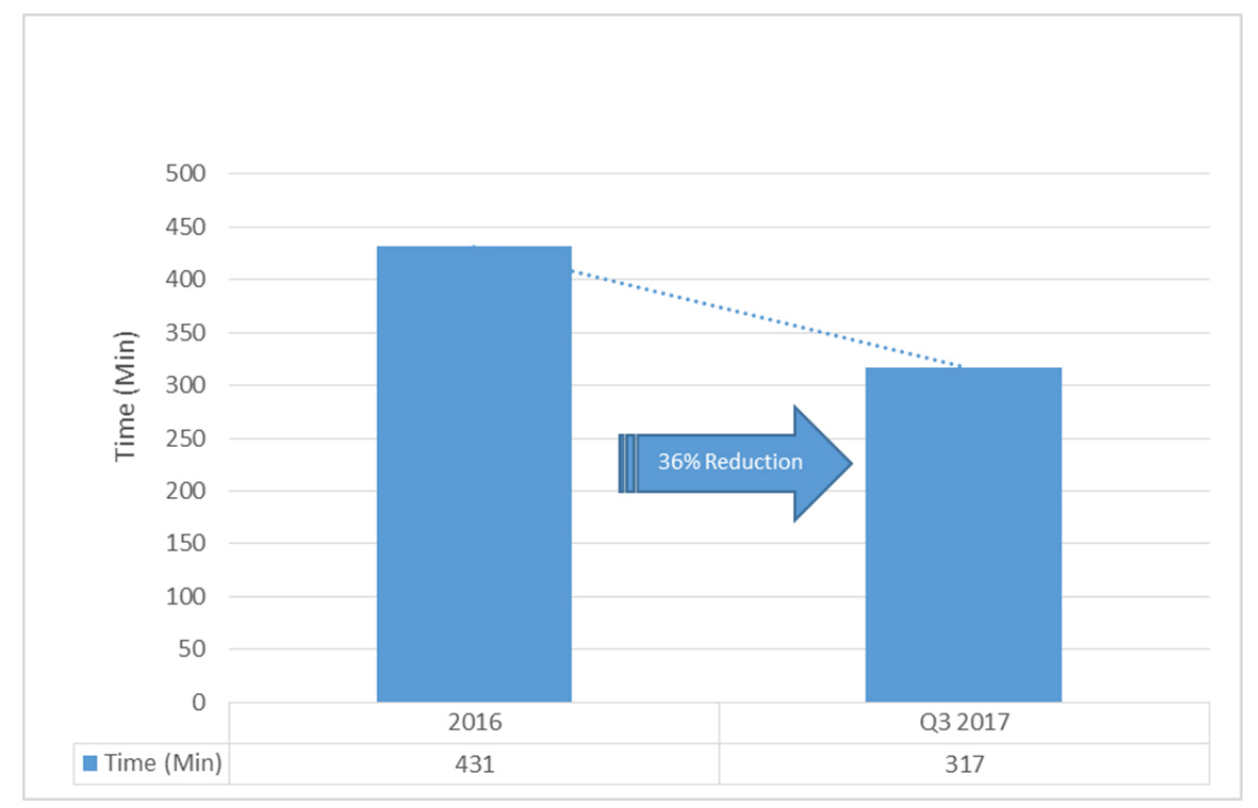

Figure 3. ED arrival to departure for psych patients discharging

*Source: CMS OP-18c - Median Time from ED Arrival to ED Departure for Discharged Patients - Psychiatric Patients 


\section{IMPLICATIONS FOR PRACTICE AND NEXT STEPS}

The impact of the actions described above was to directly influence the quality and timeliness of care for psychiatric patients in a forum that is becoming a last resort for patients seeking help. This holistic model of action is intended to reduce the LOS for patients seeking care, provide better medical management of the patients' needs, and ensure that the patients and their clinical caregivers are kept safe while doing so.

Given the limitations mentioned around the resources available in the community, the recommended next steps for Houston Methodist Hospital and the public at large are to continue efforts to expand the continuation of post-hospital-stay care offered. Expansion in resources such as additional psychiatric inpatient beds is needed as well as an influx of substance use disorder management centers in the greater Houston area. Vision exists on the furthered integration of telemedicine in the continued care of patients in the home setting. Funding to support that endeavor is desirable. Considering that mental health drugs can be costly to the patient, it is advisable to develop cheaper alternatives or programs that will assist those in need with fiscal support for their continued care. Promotion of a greater pipeline of psychiatrists available to meet the growing patient population is of critical importance. It will only be through consideration and action of these opportunities that the vicious cycle of patients unnecessarily returning to the hospital setting or landing in jail will be mitigated. There is a clear ethical responsibility of our community to address this gap in care in order to keep this patient population not only safe and secure, but promote each and every one as healthy contributors to our society. Something can and must be done.

\section{CONFlicts OF InTEREST Disclosure}

The authors declare that there is no conflict of interest.

\section{REFERENCES}

[1] Ackerman T. UTHealth gets state's go-ahead for new psychiatric hospital in Houston. Houston Chronicle. 2018 Jan 11 [cited 2018 Aug 15]. Available from: https://www.chron.com/news/hou ston-texas/article/UTHealth-gets-state-go-ahead-f or-new-psychiatric-12482221.php

[2] Texas Department of State Health Services. Texas Population, 2017 (Projections). 2014 Dec 16. [cited 2018 Aug 15]. Available from: https://www.dshs.texas.gov/chs/popdat/ST2017.shtm

[3] Jordan JR. Davis to push for mental health, parole reform in 85th Legislature. Houston Chronicle. 2016 Aug 15 [cited 2018 Aug 15]. Available from: https://www.chron.com/neighborhood/bel laire/news/article/Davis-to-push-for-mental-healt h-parole-reform-in-9893154.php

[4] Walters E. State spending more on mental health care, but waitlist grows. Texas Tribune [Internet].
2016 May 1 [cited 2018 Aug 15]. Available from: https : //www . texastribune.org/2016/05/01/despit e-state-spending-dearth-pysch-hospital-beds /

[5] American Psychiatric Association. Telepsychiatry [Internet]. c2018 [cited 2018 Aug 15]. Available from: https: //www.psychiatry .org/psychiatrists/practice/telepsychiatry

[6] Khullar D. The largest health disparity we don't talk about. New York Times. 2018 May 30 [cited 2018 Aug 15]. Available from: https://mobile.nytimes.com/2018/05/30/upshot/m ental-illness-health-disparity-longevity.html

[7] Egede LE, Acierno R, Knapp RG, et al. Psychotherapy for depression in older veterans via telemedicine: effect on quality of life, satisfaction, treatment credibility, and service delivery perception. J Clin Psychiatry. 2016; 77(12): 1704-1711. PMid:27835713 https://doi.org/10.4088/JCP.16m10951 